\title{
Ermöglicht die Flexibilisierung in der Berufsausbildung mehr Chancengerechtigkeit bei der Ausbildungsplatzvergabe? Fallstudie eines Schweizer Ausbildungsverbundes
}

\author{
Christian Imdorf, Regula Julia Leemann
}

Published as:

Flexibilisierung in der Berufsausbildung mehr Chancengerechtigkeit bei der

Ausbildungsplatzvergabe? Fallstudie eines Schweizer Ausbildungsverbundes. In:

D. Voss-Dahm, G. Mühge, K. Schmierl \& O. Struck (eds.), Qualifizierte

Facharbeit im Spannungsfeld von Flexibilität und Stabilität. Organisations- und personalpolitische Innovationen im Betrieb. Wiesbaden: VS-Verlag für

Sozialwissenschaften, 49-74.

DOI 10.1007/978-3-531-92752-7_3

Original source of publication:

http://www.springerlink.com/content/181724q17m185v00/

The final publication is available at www.springerlink.com

\section{Kontaktadresse}

Prof. Dr. Christian Imdorf

Seminar für Soziologie der Universität Basel

Petersgraben 27

4051 Basel

E-Mail: christian.imdorf@unibas.ch

Tel. +416126728 82

Fax. +41612672820 


\begin{abstract}
In diesem Beitrag wird untersucht, ob Berufslehrverbünde eine gerechtere, d.h. weniger diskriminierende Selektion von Lehrlingen in die Berufslehre ermöglichen. Berufslehrverbünde sind eine sich in der Schweiz wie auch in Deutschland und Österreich verbreitende neue Organisationsform in der Berufsbildung, bei der sich mehrere und oft kleinere Betriebe zusammenschliessen, um gemeinsam Lernende auszubilden. Im in der Schweiz geförderten Modell rekrutiert eine intermediäre Leitorganisation die Lernenden. Die Leitorganisation betreut zudem das Lehrverhältnis und platziert die Jugendlichen in der Regel jährlich neu in einem der beteiligten Ausbildungsbetriebe. Theoretisch stützt sich die Studie auf die französische Soziologie der Konventionen, welche es erlaubt, die organisationalen Motive und Mechanismen der Selektion in Ausbildungsinstitutionen und die darin sich bergenden Einfallstore für Diskriminierung zu verstehen. Auf der Basis einer Fallstudie eines mittelgrossen Lehrbetriebsverbundes wird nachgezeichnet, wie diese Organisationsform es erlaubt, im Vergleich zu einzelbetrieblichen Selektionsprozessen in Klein- und Mittelbetrieben eine gerechtere, d.h. mehr an Leistung und weniger an sozialen Merkmalen der Bewerber/innen orientierte Selektion durchzuführen.
\end{abstract}




\begin{abstract}
„Also, was die Selektion anbelangt, ich mache immer sozusagen eine Gratwanderung. Ich möchte nicht irgendwie Superstars haben, die überall eine Lehrstelle finden. (...) Ich möchte immer auch Leute nehmen, die irgendwo ein Problem haben. Und ich habe auch schon viele Vorurteile abbauen können. Dass z.B. Leute aus dem ex-jugoslawischen Raum und dunkle Menschen, dass die auch Menschen sind, das haben viele Firmen bei unserem Lehrbetriebsverbund gemerkt" (Geschäftsführer eines Ausbildungsverbunds)
\end{abstract}

\title{
$1 \quad$ Einleitung
}

Um den Mangel an betrieblichen Ausbildungsplätzen zu Beginn des 21. Jahrhunderts zu beheben und den Schwierigkeiten von Schulabgängern bei der Lehrstellensuche vorzubeugen, betreibt die Schweizer Berufsbildungspolitik seit einigen Jahren „Lehrstellenmarketing“. Der Bund fördert in diesem Rahmen so genannte Lehrbetriebsverbünde $(\mathrm{LBV})^{1}$ durch Anschubfinanzierung, Beratung und Vernetzung. Die Gesetzgebung zur Berufsbildung ${ }^{2}$ sieht neben den traditionellen Lehrbetrieben und Lehrwerkstätten seit einigen Jahren LBV als Lernorte zur Vermittlung der beruflichen Grundbildung vor. Ein LBV wird definiert als Zusammenschluss von mehreren Betrieben zum Zweck, Lernenden in verschiedenen spezialisierten Betrieben eine umfassende Bildung in der beruflichen Praxis zu gewährleisten. ${ }^{3}$

Die Bildungsbewilligung für den LBV wird einer intermediären Organisation, der so genannten Leitorganisation (LO), erteilt. Diese rekrutiert die Lernenden $^{4}$ und schliesst mit ihnen den Lehrvertrag ab, d.h. die am Verbund teilnehmenden Betriebe benötigen selbst keine Ausbildungsbewilligung mehr. Die Ausbildungsbetriebe geben damit die Verantwortung über das Ausbildungsverhältnis an die LO ab. Die Lernenden rotieren über ihre Ausbildungszeit in der Regel jährlich zwischen den Ausbildungsbetrieben (vgl. Abbildung 1). Die Ausbildungsorganisation dieses als ,Großverbund' bezeichneten Modells ${ }^{5}$ entspricht

\footnotetext{
${ }^{1}$,Lehrbetriebsverbund' ist der in der Schweiz gebräuchliche Begriff für einen Ausbildungsverbund. ${ }^{2}$ Bundesgesetz über die Berufsbildung (Berufsbildungsgesetz, BBG) vom 13. Dezember 2002 (Stand am 14. Dezember 2004)

${ }^{3}$ Art. 6 Bst. c der Verordnung über die Berufsbildung (Berufsbildungsverordnung, BBV) vom 19. November 2003 (Stand am 1. August 2009).

${ }^{4}$ Auszubildende werden in der Schweiz offiziell als ,Lernende' bezeichnet.

${ }^{5}$ In der Schweiz werden drei Organisationsmodelle von LBV unterschieden: Beim Modell ,Großverbund', auf das sich der vorliegende Beitrag bezieht, handelt es sich um das in der Schweiz offiziell geförderte Verbundsystem. Daneben gibt es zwei weiter Modelle: Im Modell ,Ergänzungsausbildung' deckt ein Lehrbetrieb den größten Teil der betrieblichen Ausbildung selber ab und schließt sich für den fehlenden Teil mit einem Partnerbetrieb oder mit einer Ausbildungsstätte zusammen. Im
} 
damit der Organisationsform des deutschen Ausbildungsvereins in Klaus Schmierls Typologie von Lernallianzen (vgl. den entsprechenden Beitrag im vorliegenden Band). ${ }^{6}$

LBV können einerseits bottom up entstehen, indem sich verschiedene Betriebe zusammenschließen, eine LO benennen und die Zusammenarbeit vertraglich regeln. Andererseits entstehen LBV auch top down, indem staatliche oder private Akteure (z.B. ein Gewerbeverband) eine LO einsetzen, welche interessierte Ausbildungsbetriebe rekrutiert.

Abbildung 1: $\quad$ Struktur eines Schweizer LBV des Typs Großverbund und Rotation der Lernenden

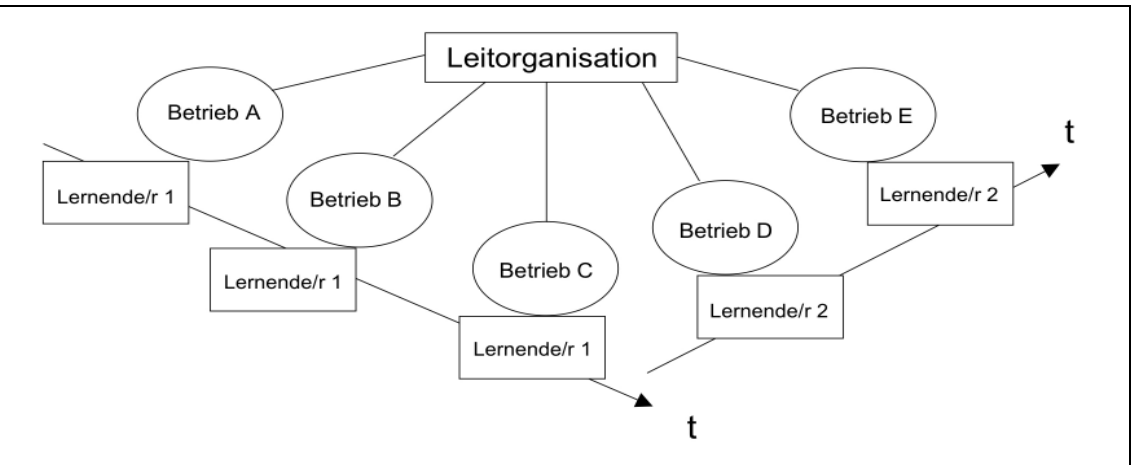

Die Förderung der Verbundausbildung durch die Schweizer Berufsbildungspolitik dient nicht nur dem gemeinnützigen Zweck, zusätzliche Ausbildungsplätze zu schaffen. Die neue Ausbildungsform ist auch eine Reaktion auf veränderte Ausbildungsanforderungen an die Betriebe: Steigende Flexibilisierungsanforderungen, die Rationalisierung der Produktionsprozesse sowie die Standardisierung der Produkte haben dazu geführt, dass manche, insbesondere kleinere Betriebe, nur noch Teile einer Ausbildung anbieten können. Die Verbundausbildung ermöglicht es solchen Betrieben, weiterhin oder neu auszubilden und damit zum beruflichen Nachwuchs der Branche und des eigenen Unternehmens beizutragen.

Modell ,Kleinverbund' schließen sich mehrere Firmen mit komplementären Tätigkeitsgebieten zusammen und decken so die betriebliche Ausbildung ab. Jede Firma kann die Funktion der Leitfirma übernehmen (DBK 2002).

${ }^{6}$ Die Entsprechung besteht insbesondere in der zentralen Zuständigkeit einer operativen Stelle für die Lehrlingsrekrutierung und die Ausbildungsorganisation, sowie für die Vertretung des Verbunds gegenüber externen Verbundpartnern und der Berufsbildungsbehörde. Gemeinsam sind den beiden Modellen auch die für die Lernenden wechselnden betrieblichen Lernorte und das damit einhergehende Rotationsprinzip. 
Ein weiteres Potenzial von LBV, welches die Berufsfachlichkeit betrifft, ist die Ausbildung für Berufe mit verschiedenen Tätigkeitsbereichen. Ein konkretes Beispiel für einen solchen Fall ist die Ausbildung zu Fachangestellten Betreuung $(\mathrm{FaBe})$, welche für Betreuungsaufgaben im Kinder-, Betagten- und Behindertenbereich qualifiziert (siehe Kapitel 3.2).

Ausbilden im Verbund ist für viele Betriebe besonders attraktiv, weil sie bei der Erbringung von Ausbildungsleistungen durch die LO vielseitig entlastet und von der Verantwortung für das Ausbildungsverhältnis weitgehend befreit werden. Bei den Dienstleistungen der LO, an denen sich die Verbundbetriebe finanziell beteiligen, umfassen die Administration des Ausbildungsverhältnisses, die Betreuung von Verbundbetrieben und Lernenden (u.a. Beratung und Entlastung bei schulischen oder betrieblichen Ausbildungsproblemen, Kontrolle der Lernzielerreichung), die Übernahme von Ausbildungsaufgaben, sowie die Organisation der Rotation. Nicht zuletzt rekrutiert die LO die Lernenden des Gesamtverbundes und entlastet damit die Einzelbetriebe von der zeitaufwändigen und risikoreichen Auswahl neuer Auszubildender. Für diese Rekrutierungsaufgabe sind insbesondere kleinere Ausbildungsbetriebe oft schlecht gerüstet, da es ihnen an professionellem Wissen, zuverlässigen Selektionsinstrumenten und Zeit für die Selektionsaufgabe fehlt (Imdorf 2010a).

LBV leisten möglicherweise auch einen spezifischen Beitrag zur beruflichen Stabilisierung. Das erweiterte Betreuungssystem der Lernenden mit einer intensiveren Betreuung, individueller Lernförderung und gezieltem Eingreifen bei Lernproblemen könnte zum einen Lehrabbrüche vermeiden helfen. Unseres Wissens gibt es dazu aber für den deutschsprachigen Raum keine verlässlichen Angaben. Andererseits erleichtert die neue Ausbildungsorganisation den Übergang in die Erwerbstätigkeit nach Abschluss der Ausbildung. Gemäss einer BIBB-Befragung von Ausbildungsabsolventen kann in Deutschland von grösseren Übernahmechancen nach einer absolvierten Verbundausbildung ausgegangen werden (BMBF 2004: 145). Auch eine Schweizer Studie zum Übernahmeverhalten belegt, dass die Verbleibquoten bei Mitgliedsfirmen von externen Ausbildungszentren im Vergleich zu traditionellen Ausbildungsbetrieben grösser ist (Schweri et al. 2003: 188f.).

Der vorliegende Beitrag untersucht die Auswirkungen der Organisationsform eines LBV (Modell Großverbund) auf die Auswahl der Lernenden. Von besonderem Interesse sind dabei die Konsequenzen des ,Outsourcings' der Lehrlingsselektion aus den Betrieben an die LO auf die chancengerechte Vergabe von Ausbildungsplätzen. Die Frage der Chancengleichheit bei der Lehrlingsauswahl wurde im Zusammenhang mit Lehrbetriebsverbünden bisher kaum gestellt. Die bildungspolitisch motivierte Initiative, mit LBV zusätzliche Ausbildungsplätze 
zu schaffen, fragt in der Regel nicht danach, ob alle Ausbildungsnachfrager davon in gleichem Maß profitieren.

Da zur Lehrstellenvergabe in LBV bisher kaum wissenschaftliche Erkenntnisse vorliegen, werden in einem kurzen Forschungsüberblick zunächst die Auswahlkriterien resümiert, welche die Selektion von Lernenden in traditionellen Ausbildungsbetrieben anleiten. Mit Bezugnahme auf die französische Soziologie der Konventionen wird sodann ein theoretischer Rahmen skizziert, der sich neben der Analyse von Selektionsprozessen in Einzelbetrieben auch dazu eignet, die Lehrlingsauswahl eines LBV sowie das Phänomen der Diskriminierung bei der Ausbildungsstellenvergabe zu verstehen. Im empirischen Teil wird anhand einer explorativen Fallstudie rekonstruiert, wie ein Ausbildungsverbund, der von staatlicher Seite auch den Auftrag erhalten hat, die Integration benachteiligter Schulabgänger zu verfolgen, selektiert. Es soll insbesondere geklärt werden, wie es dem untersuchten LBV gelingt, sein soziales Vorhaben in der Kooperation mit Ausbildungsbetrieben, die vom sozialen Auftrag des Verbundes kaum Kenntnis haben, organisatorisch umzusetzen. Der Schlussteil resümiert die Hypothesen generierende Fallstudie und verweist auf offene Forschungsfragen.

\section{Forschungsstand und Theorie}

\subsection{Auswahlkriterien bei der Selektion von Lernenden}

Aus der deutschen und schweizerischen Evaluationsforschung zu Ausbildungsverbünden liegen zur Frage der Rekrutierung und Selektion bisher nur spärliche Hinweise vor. Gemäss einer Evaluation des Schweizer Bundesamts für Berufsbildung und Technologie (BBT 2008: 11) rekrutiert in drei Viertel aller Fälle die LO die Lernenden alleine. Letztere werden sodann auf die Verbundbetriebe verteilt, die meist ein Vetorecht besitzen. Der Rekrutierungspool der Lernenden hängt von der Ausrichtung des LBV ab. Es gibt Verbünde, welche die Lernenden ausschliesslich direkt nach der obligatorischen Schulzeit anstellen, oder aber solche, die Absolventen des Übergangssystems den Vorrang geben (ebd.: 9). Verbünde ermöglichen dabei Ausbildungsangebote und Förderung sowohl für leistungsschwache als auch für leistungsstarke Jugendliche (Walther \& Renold 2005). Gemäss einer Befragung aus Deutschland besassen deutsche Verbundabsolventen deutlich öfter einen Hauptschulabschluss und seltener die Hochschulreife (Abitur) als die Lernenden einer Vergleichsgruppe in traditionellen Betriebsausbildungen (BMBF 2004: 144f). Dies gibt Anlass zur Annahme, dass Ausbildungsverbünde insbesondere auch benachteiligten Schulabgängern zu einer Lehrstelle verhelfen können. 
Während die konkreten Selektionsverfahren und -kriterien von Ausbildungsverbünden bisher aber kaum erforscht sind, liegen zur Auswahl in traditionell organisierten Ausbildungsbetrieben Erkenntnisse aus mehreren Untersuchungen vor. Diese heben zum einen die große Bedeutung von Schulleistungen in Großbetrieben (Moser 2004) sowie generell den ,,persönlichen Eindruck“ der Bewerber hervor (Schmid \& Storni 2004; Stalder 2000). Eine eigene Untersuchung zur Lehrlingsauswahl in kleinen und mittelgroßen Betrieben (KMU) hat dagegen gezeigt, dass die Vergabe von Ausbildungsplätzen primär einer Logik der „Betriebstauglichkeit“ folgt, die versucht, Problemrisiken für den eigenen Betrieb im Zusammenhang mit Auszubildenden frühzeitig vorzubeugen (Imdorf 2008). Schulleistungen werden bei der Selektion in KMU mitberücksichtigt, um abzuschätzen, ob die Minimalanforderungen der Berufsschule an die Lernenden erfüllt sein werden (Imdorf 2009).

Betriebe suchen aber nicht nur nach Auszubildenden, deren Ausbildung möglichst wenige Probleme verursacht. Auch der Auswahlprozess selbst darf nicht zu viele Ressourcen binden. Im Gegensatz zu kleinen und mittleren Unternehmen kommt den Schulqualifikationen in Großbetrieben aus dieser Perspektive eine erhöhte vorselektive Bedeutung zu, da hier der Bewerberandrang größer ist und die Betriebe auf kostengünstige Formen der frühen Auslese angewiesen sind. Schulische Leistungsmerkmale (Schultyp, Schulnoten, Leistungstests) werden entsprechend in Abhängigkeit des Bewerberdrucks variabel eingesetzt, um den Auswahlprozess insbesondere bei hohen Bewerberzahlen zu „objektivieren“ (Sehringer 1989: 160). Dagegen führen bei der Selektion in KMU die Selektionskriterien der sozialen Passung, der Kontrollier- und Führbarkeit, sowie der Kundenpräferenzen bereits im Rahmen der Vorselektion dazu, dass diskriminierende Kategorien wie Ausländerstigma, Geschlecht und Alter die Ausbildungsplatzvergabe frühzeitig mitstrukturieren (Imdorf 2010a, 2010c). In Großbetrieben, die einen höheren Formalisierungsgrad bei ihrer Ausbildungsplatzvergabe aufweisen, dürften ausländische Jugendliche hingegen vor derartigen Formen der Diskriminierung durch den Betrieb eher geschützt sein (Seibert, Hupka \& Imdorf 2009).

\subsection{Selektion in Ausbildungsverbünden aus konventionensoziologischer Sicht}

Selektionsprozesse in Ausbildungsverbünden zu untersuchen verlangt nach einer Theorie, mit der sich zum einen Personalselektion und Diskriminierung verstehen lassen, und die zum anderen auch der komplexen Organisationsstruktur und Koordinationsformen von LBV gerecht werden kann. Die französische Soziologie der Konventionen vermag beide Ansprüche einzulösen. Es handelt sich dabei um einen transdisziplinären, sowohl in den französischen Wirtschaftswissen- 
schaften als auch in der französischen Soziologie etablierter Ansatz, der auf der soziologisch-pragmatistischen Theorie von Boltanski und Thévenot (2007) gründet und verschiedene Bindestrichsoziologien (u.a. die Wirtschafts-, Arbeits- und Organisationssoziologie) auf der Grundlage einer pragmatischen Handlungstheorie integriert (Diaz-Bone 2009). Im Zentrum steht die Beobachtung und Analyse der Formen, wie sich Personen untereinander oder mit ihrer Umgebung im konkreten Handeln abstimmen, sowie die Konventionen, die zur Bildung der Gemeinsamkeit der Perspektiven beitragen, um dieses Handeln koordinieren und rechtfertigen zu können (Dodier 2010). Unter Konventionen werden kollektiv etablierte kulturelle Formen dessen verstanden, wie Beziehungen zwischen mehreren Akteuren gestaltet, evaluiert und legitimiert werden. Die Bewertung eines Akteurs (bzw. dessen ,Qualität', vgl. folgende Beispiele) aufgrund unterschiedlicher Konventionen verweist dabei auf Koordinationszusammenhänge mit unterschiedlichen Ordnungs- und Gerechtigkeitsprinzipen, die von Boltanski und Thévenot (2007: 183) als ,Welten ' bezeichnet werden (zur Anwendung des Welten-Konzepts auf die Ausbildungsplatzvergabe vgl. Imdorf, 2008).

Als zentrale Konventionen des Wirtschaftssystems, nicht zuletzt auch der Einstellung neuer Mitarbeiter und Auszubildender, gelten die industrielle, die marktwirtschaftliche, die familienweltliche sowie die Netzwerk bezogene Konvention (wir beziehen uns nachfolgend auf die Skizzierung dieser Konventionen bei Diaz-Bone 2009: 242f). Die industrielle Konvention folgt dem Prinzip der mittel- und langfristigen Handlungsplanung, wobei Ressourcen produktiv genutzt werden sollen. Die Handlungsabläufe orientieren sich entsprechend an einer effizienten Arbeitsorganisation. Arbeitsmethoden, Arbeitsbeziehungen und Produkte sind standardisiert und die Akteure (bzw. ihre ,Qualität') werden danach beurteilt, wie produktiv ihre Beteiligung an der Produktherstellung ist. In dieser ,industriellen Welt' gilt das Gerechtigkeitsprinzip Leistung (Meritokratieprinzip). Bei der Selektion von Auszubildenden ist von Bedeutung, dass sie die künftigen Produktionsabläufe aufgrund ihrer beruflichen und schulischen Fähigkeiten möglichst unterstützen und nicht stören werden.

Die marktwirtschaftliche Konvention hebt dagegen in einer kurzfristigen Zeitperspektive das Beziehungsprinzip des Preises und der Konkurrenz hervor: Bei der Kooperation von LO und Ausbildungsbetrieben eines LBV können sich u.a. ökonomische Kosten-Nutzen-Kalküle der Netzwerkpartner als relevant erweisen. Die Art der Beziehung zwischen den Akteuren ist strategisch und ihre Koordination orientiert sich opportunistisch an der Marktlage. Ein Akteur selbst erhält ,Qualität' entweder als solvente Kaufkraft oder durch die Bereitstellung eines nachgefragten Gutes mit knappem Angebot (als Verkäufer). Auf die Auswahl von Auszubildenden übertragen bedeutet diese Marktlogik, dass ein künftiger Lernender in der , marktförmigen Welt' mit seinem Arbeitskraftangebot den 
Vertrieb eines Produktes oder einer Dienstleistung möglichst fördert und nicht hemmt. Insbesondere im Direktkontakt mit der Kundschaft erhält der Lernende damit einen eigentlichen Marktwert, was dazu führen kann, dass aufgrund von Präferenzen der Kunden bei der Personalselektion soziale Merkmale relevant werden (Becker 1971: 75f).

Basierend auf der familienweltlichen Konvention erlangt ein Akteur, Qualität', wenn ihm aufgrund von ,sozialer Nähe', Bekanntschaft, Verwandtschaft und persönlichen Beziehungen Vertrauen entgegengebracht wird. Die Wertigkeit der Auszubildenden misst sich entsprechend an deren Vertrauenswürdigkeit und Zuverlässigkeit, an Loyalität und ,Charakter'. Im Gegensatz zu den zwei erstgenannten Konventionen ist die Zeitperspektive der familienweltlichen Konvention eher auf Tradition und damit auf die Vergangenheit ausgerichtet. Auf diese Konvention berufen sich Ausbildungsbetriebe bei der Selektion in der , häuslichen Welt', wenn sie einen Anspruch auf Lernende geltend machen, die zur betriebsinternen Belegschaft möglichst sozial passen. Die Ausländer-Diskriminierung bei der Auswahl von Auszubildenden erweist sich in besonderem Masse als die Folge einer familienweltlichen Selektion (Imdorf 2008).

Während sich in KMU Prozesse der Ausbildungsplatzvergabe mit den drei skizzierten Konventionen weitgehend verstehen lassen (Imdorf 2010c), legt es die Netzwerkstruktur von Ausbildungsverbünden nahe, auch die sog. Netzwerkkonvention bei der Untersuchung von Selektionsprozessen in LBV zu berücksichtigen. Diese von Boltanski und Chiapello (2003) rekonstruierte Konvention weißt Akteuren dann ,Qualität' zu, wenn sie Netzwerke bilden, über Flexibilität, Mobilität und Risikobereitschaft verfügen und sich als ,projektfähig' erweisen. Im Unterschied zur familienweltlichen Beziehungsform werden Beziehungen in der ,projektförmigen Welt' flexibel und befristet gelebt, d.h. die sozialen Bindungen und Freundschaften sind in der Zeitperspektive dieser Konvention nur von relativer bzw. mittlerer Dauer. Bei der Vergabe von Ausbildungsplätzen für Verbundausbildungen könnte die Netzwerkkonvention daher bedeutsam sein, da den Lernenden im Rahmen ihrer jährlichen Rotation zwischen den Ausbildungsbetrieben möglicherweise solche projektförmige ,Qualitäten' abverlangt werden. Uns sind bisher keine Forschungsresultate bekannt, wonach die betriebliche Flexibilitätserwartung mit diskriminierenden (z.B. ethnisierenden) Kategorien verbunden wird.

Aus Sicht des Ausbildungssystems gilt es schließlich auch die von Boltanski und Thévenot (1999: 372) propagierte ,staatsbürgerliche Welt' zu berücksichtigen, um die Ausbildungsplatzvergabe von LBV zu verstehen. Die entsprechende Konvention gründet auf einem Kollektivinteresse und reklamiert einen Gesellschaftsvertrag, der das eigensüchtige Interessehandeln der Betriebe durch ein Bekenntnis zur Wahrung des sozialen Friedens einschränkt (Imdorf 2008: 126). 
Bei der Gestaltung von Beziehungen rückt das Prinzip der Chancengleichheit in den Vordergrund. Die staatsbürgerliche Norm erfordert von den Betrieben zuallererst die Einhaltung des Gesetzes. Da der Staat aber im Unterschied zur Gestaltung des Ausbildungsverhältnisses die betriebliche Lehrlingsauswahl kaum reguliert, vermag die staatsbürgerliche Konvention die Vergabe von Ausbildungsplätzen in der Regel nur schwach zu strukturieren. Sie dürfte jedoch für die Lehrstellenvergabe in jenen Ausbildungsverbünden relevant werden, die staatlich (mit)initiiert wurden oder durch die öffentliche Hand mitsubventioniert werden. Unter solchen Bedingungen hat ein LBV in der Regel einen staatsbürgerlichen Auftrag zu erfüllen, der fordert, auch benachteiligte Gruppen bei der Lehrlingsrekrutierung mit zu berücksichtigen.

Die skizzierten Konventionen können als wesentliche Beziehungs- und Koordinationsprinzipien im Kontext eines Ausbildungsverbundes betrachtet werden, welche die Abstimmung der gegenseitigen Erwartungen und Anforderungen zwischen den Akteuren LO, Ausbildungsbetriebe und Lernende gewährleisten und die Evaluation der ,Qualität' der Akteure (zukünftige Lernende) leiten. Als Äquivalenz- und Rechtfertigungsprinzipien dienen sie gleichzeitig auch zur Begründung der Selektion neuer Auszubildender.

Auch das Phänomen der Diskriminierung lässt sich konventionensoziologisch verstehen, wobei es zwei Prozesse zu unterscheiden gilt, die unabhängig voneinander zu organisationaler Diskriminierung führen (vgl. ausführlich Imdorf 2010a). Eine Lehrlingsauswahl erzeugt zum einen Diskriminierung, wenn der betriebliche Selektionsprozess nicht primär durch die Leistungsanforderungen der industriellen Welt geleitet wird. Meritokratische Leistungsgerechtigkeit ist dann nicht mehr gegeben, wenn sich bei der Personalauswahl eher die Konventionen und die darauf gründenden Erwartungen der häuslichen und der markförmigen Betriebswelt durchsetzen, also die Kalküle und Motive der betrieblichen Sozialintegration bzw. der Kundenbindung. Zum anderen kann Diskriminierung auch aus einem ,Messproblem' bei der Selektion erwachsen. Erfolgreiche Bewerber müssen ihre ,Qualität' in multiplen betrieblichen Welten unter Beweis stellen und dazu zahlreiche 'Bewährungsproben' bestehen (für empirische Beispiele vgl. Imdorf 2008). Zu Diskriminierung kommt es im Rahmen solcher Bewährungsproben dann, wenn die betriebliche ,Qualität' der Stellenbewerber aufgrund verzerrender Gruppenmerkmale (z.B. Geschlecht, Alter) bzw. aufgrund sozialer Zuschreibungsprozesse beurteilt wird, um das Selektionsverfahren zu beschleunigen und kostengünstig zu halten (Eymard-Duvernay \& Marchal 1997; Jewson \& Mason 1986). Damit sind in Abgrenzung zu den Motiven der Selektion mit Bezugnahme auf Reskin (2003) die Mechanismen der Diskriminierung angesprochen. Erst spezifische Formen der Organisation der Auswahl erlauben es den Personalverantwortlichen, ihre diskriminierenden Ste- 
reotype in den Selektionsprozess einzubringen. Auch die Durchsetzung der diskriminierenden Selektionskriterien der häuslichen oder der marktförmigen Welt erfordert ein Selektionsverfahren, welches Diskriminierung zulässt. Ein oft für Kleinbetriebe charakteristischer Einstellungsprozess, der auf sozialer Netzwerkrekrutierung basiert, erleichtert es beispielsweise der häuslichen Welt, ihr Selektionskriterium der sozialen Passung durchzusetzen (Jenkins 1984).

Vor dem Hintergrund dieser theoretischen Überlegungen soll nachfolgend zum einen der Frage nachgegangen werden, welche Konventionen die Auswahl von Lernenden in einem Ausbildungsverbund maßgeblich strukturieren (Frage nach den Motiven des Auswahlentscheides). Zum anderen soll analysiert werden, inwiefern im Unterschied zu herkömmlichen Ausbildungsbetrieben die Organisation der Lehrlingsauswahl eines LBV besser zu verhindern mag, dass sich diskriminierende betriebliche Erwartungen bei der Selektion durchsetzen (Frage nach den Mechanismen der Auswahl). Empirisch werden diese zwei Forschungsfragen im Rahmen einer Fallstudie erörtert. Konkret soll untersucht werden, welche Faktoren es einem ausgewählten Ausbildungsverbund ermöglichen, seinem Auftrag der Integration von benachteiligten Schulabgängern nachzukommen und benachteiligte Jugendliche an Verbundbetriebe zu vermitteln, die selbst kaum Kenntnis von diesem Sozialauftrag haben. In diesem Zusammenhang interessiert, wie sich die netzwerkförmige Struktur der Ausbildungsorganisation eines LBV auf die Selektion von Auszubildenden auswirkt, d.h. welches seine spezifischen Motive und Mechanismen der Selektion sind. 


\section{Fallanalyse: Methode und Portrait des ausgewählten Lehrbetriebverbundes}

\subsection{Methode}

Um die Forschungsfragen zu beantworten, wurde eine Hypothesen generierende, sekundäranalytische Fallstudie eines LBV durchgeführt. Eine Einzelfallstudie ist dem Forschungsvorhaben angemessen, da es sich bei einem LBV um einen kritischen Fall (Yin 2009: 47) einer Ausbildungsorganisation hinsichtlich der Selektionsprozesse in die duale Berufsausbildung handelt. Inwiefern sich die bekannten Unterschiede in den Selektionskriterien von Gross- gegenüber Kleinbetrieben auf einen Ausbildungsverbund übertragen lassen, ist unklar, denn ein LBV weißt hinsichtlich seiner selektionsrelevanten Strukturmerkmale eine interessante Kompromissform von klein- $u$ nd grossbetrieblichen Merkmalen auf. Zum einen sind im untersuchten Fall neben einigen wenigen Grossbetrieben vor allem Klein- und Kleinstbetriebe als Verbundbetriebe vernetzt. Zum anderen ist die durch die LO abgedeckte Personaladministration und Ausbildungsbetreuung der Ausbildungsorganisation in Grossunternehmen nicht unähnlich. Auch dort werden Lernende zentral rekrutiert und verwaltet, um dann ihre Ausbildung in verschiedenen Abteilungen des Grossunternehmens zu absolvieren. Im Unterschied zu sowohl großen wie kleinen Lehrbetrieben liegt die Ausbildungsverantwortung in einem Ausbildungsverbund jedoch nicht bei den Betrieben selbst, sondern bei der LO. Am Fall eines exemplarischen LBV soll daher geklärt werden, in welchem Verhältnis die bekannten Selektionsmerkmale von Klein- und Grossbetrieben auf diese neue Ausbildungsform übertragbar sind, bzw. inwiefern es die bisherigen wissenschaftlichen Erkenntnisse zur Ausbildungsplatzvergabe zu erweitern gilt, um auch die Selektion für eine Ausbildung im Verbund zu verstehen.

Da das Datenmaterial aus einer Evaluationsstudie stammt und im Hinblick auf die eigenen Fragestellungen reanalysiert wurde, handelt es sich bei der Fallstudie um eine Sekundäranalyse. Im Rahmen der Evaluation ${ }^{7}$ wurden u.a. die Themen der Selektion und der Rotation in halbstandardisierten Interviews differenziert erfragt, wobei innerhalb des untersuchten Ausbildungsverbundes heterogene Akteursperspektiven berïcksichtigt wurden. Das Datenmaterial umfasst total neun Interviews: zwei Interviews mit dem Geschäftsführer der LO sowie einer Mitarbeiterin, welche für die Administration der Lernenden zuständig ist und bei der Vorselektion mitgeholfen hat; zwei Interviews mit insgesamt drei sog. Berufsbildungskoordinatorinnen und -koordinatoren (diese ,Lehrmeister' im

${ }^{7}$ Die Evaluationsstudie wurde durchgeführt von der Autorin Regula Julia Leemann, gemeinsam mit Dr. Simone Berweger, Pädagogische Hochschule Zürich. 
juristischen Sinne, die über eine Ausbildungsberechtigung verfügen und in der LO die Betreuung der Lernenden und die Ausbildungskontrolle übernehmen, werden nachfolgend als ,BB-Koordinator(en)' bezeichnet); schließlich fünf Interviews mit insgesamt acht Ausbildner und Ausbildnerinnen, die in verschiedenen Verbundbetrieben für die Anleitung und Ausbildung der Lernenden zuständig sind (in zwei Behindertenheimen, zwei Kindertagesstätten, zwei Altersheimen, sowie in einem Ausbildungsbetrieb ${ }^{8}$ für Informatikerinnen und Elektropraktiker).

Damit gestattet das Datenmaterial der Evaluationsstudie ein exploratives Einzelfalldesign mit multiplen Analyseeinheiten (LO und Verbundbetriebe, zum entsprechenden embedded single-case design vgl. Yin 2009: 47). Die verschiedenen Sichtweisen auf das ,Selektionsgeschäft' erlauben es, die Ausbildungsplatzvergabe in einem LBV angemessen zu verstehen. Mittels einer qualitativen Analyse des transkribierten Interviewmaterials wurden zum einen das Selektionsverfahren (Mechanismen) und zum anderen die Konventionen (Motive) rekonstruiert, welche der Rekrutierung und Selektion der Lernenden zugrunde liegen.

\subsection{Portrait des untersuchten Ausbildungsverbundes}

Der analysierte LBV wurde zunächst als Reaktion auf die Lehrstellenkrise der späten 1990er Jahre in der Deutschschweiz durch ein städtisches Laufbahnzentrum als Verein initiiert. Zu Beginn wurden nur Kauffrauen und -männer ausgebildet. In den Folgejahren wurde die Anzahl der Lernenden und der Verbundbetriebe stetig erhöht und das berufliche Angebot ausgeweitet. Im Jahr 2006 wurde der Verein mit Hilfe einer kommunalen Kapitaleinlage in eine gemeinnützige Stiftung umgewandelt und von der Stadtverwaltung abgekoppelt. Ein zentraler Stiftungszweck besteht gemäß den Stiftungsstatuten in der Integration von sozial benachteiligten Jugendlichen (Frauen, Migranten, gesellschaftlich benachteiligte Gruppen) ins Berufsleben. Die Integration soll durch den Ausbau des Lehrstellenangebotes und die Zusammenarbeit mit privaten und öffentlich-rechtlichen Betrieben erfolgen. Die Stiftung finanziert sich neben den Beiträgen der Ausbildungsbetriebe u.a. mit kommunalen Geldern, in der Pilotphase mit Bundesmitteln sowie mit gezielt akquirierten finanziellen Spenden von privaten Firmen. Zum Befragungszeitpunkt 2007/2008 bot der Verbund pro Jahr bis zu 180 Ausbildungsstellen in 130 Betrieben für die Berufe Kaufmann/Kauffrau, Informatiker/in, Elektropraktiker/in, sowie Fachangestellte/r Betreuung an. Bei den Ver-

${ }^{8}$ Es handelt sich hier um einen Spezialfall eines Verbundbetriebs: Der LBV schickt die ausgewählten Auszubildenden für die Elektropraktiker- und für die Informatiker-Ausbildung im ersten Ausbildungsjahr in eine kostenpflichtige Lehrwerkstätte, die von diesem Partnerbetrieb angeboten wird. 
bundbetrieben handelt es sich auch um Klein- und Kleinstbetriebe, die ohne die Verbundlösung keine Möglichkeit hätten oder nicht bereit wären, selbst auszubilden. In besonderem Maße auf die Verbundausbildung angewiesen ist zudem die neue dreijährige Ausbildung zum/zur Fachmann/-frau Betreuung (FaBe) mit generalistischem Profil, im Rahmen derer die Lernenden je ein Ausbildungsjahr in einer Institution für Kinder-, Betagten- und Behindertenbetreuung absolvieren.

Bemerkenswerterweise wird der Stiftungszweck in der Selbstdarstellung des Verbundes gegenüber potenziellen neuen Ausbildungsbetrieben nicht explizit hervorgehoben. Der LBV präsentiert auf seiner Webseite vielmehr die betrieblichen Vorteile einer Verbundausbildung (u.a. Ausbildungsmöglichkeit für spezialisierte Betriebe, Produktivität des Lernenden, Möglichkeit der späteren Übernahme des Lernenden, tiefe Lehrabbruchquote). Zudem wird den potenziellen Verbundbetrieben ein berufsbezogenes Auswahlverfahren im Hinblick auf das betriebliche Stellenprofil versprochen.

\section{Konventionensoziologische Analyse des Selektionsprozesses}

Der Rekrutierungspool der Lehrstellenbewerber ergibt sich zum einen durch die Inserierung der Ausbildungsstellen im Internet über den kantonalen Lehrstellennachweis. Ergänzt werden diese Bewerbungen durch Zuweisungen eines mit dem LBV vernetzten Berufsinformationszentrums, welches dem Ausbildungsverbund bei der Lehrstellensuche erfolglos gebliebene, aber als , ausbildungstauglich' eingeschätzte Schulabgänger vermittelt. Beide Gruppen stellen sich dem gleichen Selektionsprozess.

Das aus den Interviews rekonstruierbare Selektionsverfahren des untersuchten LBV gliedert sich im Anschluss an die Rekrutierung in vier Stufen: Vorselektion der Bewerbungsdossiers, interne Eignungstests, Bewerbungsgespräch, sowie Kurzpraktikum im Betrieb. Im Folgenden werden die einzelnen Verfahrensschritte beschrieben und die darin geprüften Selektionskriterien konventionensoziologisch interpretiert.

\subsection{Vorselektion der Bewerbungsdossiers (durch LO)}

In der Vorselektion werden die schriftlichen Bewerbungen zunächst durch die LO einer „Grobselektion“ (BB-Koordinator Informatik) unterzogen. Ausschlaggebend sind der in den Bewerbungsunterlagen dokumentierte absolvierte Schultyp und die Schulnoten der Bewerber, sowie, für eine kaufmännische Ausbildung, die Resultate externer Leistungstests, welche bei spezifischen Anbietern gegen privat zu erbringende Kosten absolviert werden müssen. Im Zentrum der 
Beurteilung steht die Frage, ob der schulische Leistungsstand den Anforderungen der Berufsschule eines Ausbildungsberufs angemessen ist. Der BBKoordinator für die schulisch eher anspruchsvolle Informatikausbildung meinte: „Es gibt halt einfach Bewerbungen, da muss man sagen, da ist es von der Ausbildung, vom Werdegang her, gar nicht möglich, dass das geht. Also werden die jetzt sicher einmal gerade aussortiert“. Den schulischen Erfordernissen liegt dabei eine auf betriebliche und schulische Nützlichkeit bzw. Effizienz ausgerichtete Konvention zugrunde: ein Ausbildungsverhältnis mit einer Person zu besetzen, die absehbar an den Leistungsanforderungen der Berufsschule scheitern könnte, würde einer betrieblichen Fehlinvestition gleichkommen.

In Anbetracht des kommunalen Auftrags des LBV, auch benachteiligten Schulabgängern Ausbildungsplätze zu ermöglichen, berücksichtigt die LO bei der Vorselektion auch die staatsbürgerliche Konvention. So werden insbesondere Bewerbungen von solchen Jugendlichen einbezogen, die auf der Lehrstellensuche bereits viele Absagen erhalten haben. In den Augen des BB-Koordinators FaBe erschwere dies die Selektionsaufgabe allerdings: „Wir müssen einerseits gute Lernende haben, die dann auch erfolgreich sind. Aber wir haben von der Stadt auch noch einen Sozialauftrag zu erfüllen“. Offensichtlich konfligieren die Ansprüche der industriellen Welt (gute Schulleistungen) mit jenen der staatsbürgerlichen Welt (soziale Integration). Hinsichtlich der schulischen Selektionslogik muss entsprechend ein Kompromiss erzielt werden, um das Integrationsziel der Ausbildungsorganisation nicht $\mathrm{zu}$ verfehlen. Gemäß den Aussagen des BBKoordinators $\mathrm{FaBe}$ wird dieser Kompromiss realisiert, indem bei der Vorselektion zu je einem Drittel schulisch „sehr gute, gute sowie schwache“ Jugendliche berücksichtigt werden.

Bei Jugendlichen, die sich auf den Ausbildungsberuf Fachmann/-frau Betreuung bewerben, wird schliesslich von Angaben zu bisher absolvierten Praktika, Freizeitbeschäftigungen oder den beruflichen Tätigkeiten der Eltern im Bewerbungsdossiers auf berufsrelevante Sozialkompetenzen geschlossen, welche ebenfalls einen vorselektiven Charakter haben. Die als Selektionskriterium für Sozialberufe verlangten Sozialkompetenzen verweisen auf die Erfordernisse beruflicher Dienstleistungsbeziehungen, denen ein Kompromiss von marktwirtschaftlicher und - je nach Zeithorizont der Sozialbeziehung - häuslicher bzw. projektförmiger Beziehungslogik zugrunde liegt (Imdorf, 2008: 156f).

Hinsichtlich der Vorauswahl des untersuchten LBV fällt auf, dass die diskriminierungsanfälligen betriebsseitigen Kriterien der sozialen Passung (etwa Herkunft oder Geschlecht) im Unterschied zu den aus Klein- und Mittelbetrieben bekannten frühen Selektionskriterien keine Rolle spielen. 


\subsection{Testhalbtag' (interne Eignungstests der LO)}

Die aufgrund der schriftlichen Bewerbungsdossiers ausgewählten Kandidaten haben sich sodann im Rahmen eines „Testmorgens oder -nachmittags“ diversen internen Eignungstests zu unterziehen. Zur Prüfung werden ein Interessenstest des Laufbahnzentrums sowie der Intelligenztest PSB-R-6-13 (Prüfsystem für Schul- und Bildungsberatung für 6. bis 13. Klassen) eingesetzt. Die Kandidaten müssen zudem einen Aufsatz zu den Themen ,Wer bin ich? - Ich in fünf Jahren - Meine Familie - Weshalb gerade dieser Beruf?“ (BB-Koordinator FaBe) schreiben. Die Interviews mit den BB-Koordinatoren zeigen, dass die internen Tests neben Hinweisen auf das berufliche Interesse in erster Linie den Zeck haben, die als relativ unzuverlässig beurteilten schulischen Leistungsindikatoren des Bewerbungsdossiers mit einem testpsychologisch ermittelten Leistungsmaß zu erhärten bzw. zu ergänzen. Damit soll eine schulische Vergleichbarkeit zwischen den Bewerbern ermöglicht (,Wir müssen einfach eine Basis haben, damit wir die Bewerber vergleichen können“, BB-Koordinator FaBe) sowie eine gewisse Prognose bezüglich zukünftiger schulischer Leistungsfähigkeit erreicht werden (, ... und auch prognostizieren können (...), dass sie [die Lernenden] auch erfolgreich eine Lehre durchführen können“, BB-Koordinatorin FaBe).

Bei den internen Tests handelt es sich um Bewährungstests, denen eine industrielle Konvention zugrunde liegt, denn sie sind standardisiert, produzieren vergleichbare Resultate auf Grund ,harter Facts', und suggerieren schulische Planbarkeit. Wie schon die Selektionskriterien der Vorselektion verhindern auch diese Bewährungstests der industriellen Welt, dass sich im Auswahlverfahren die diskriminierenden Kalküle der häuslichen und marktförmigen Welt der Ausbildungsbetriebe frühzeitig durchsetzen.

\subsection{Persönliches Vorstellungsgespräch (LO)}

Bewähren sich die Bewerber im Rahmen des ,Testhalbtags', werden sie zu einem Vorstellungsgespräch mit Vertretern der LO eingeladen. Während im Rahmen einzelbetrieblicher Vorstellungsgespräche in der Regel abgeklärt wird, ob ein Kandidat in die häusliche Welt des Unternehmens passt (Jenkins 1984), erweist sich das Vorstellungsgespräch im Rahmen der Ausbildungsplatzvergabe des untersuchten LBV neben der Abklärung der Motivation (,kann der Jugendliche schon deutlich machen, dass er und warum er diesen Beruf lernen will?") als Bewährungsprobe für die Passung ins System des Ausbildungsverbundes. Abgeklärt wird ,die Flexibilität, dass jemand auch in der Lage ist, an die verschiedenen Orte zu gehen“, wie es der Geschäftsführer formuliert. Die BBKoordinatoren sehen es denn auch als eine ihrer „Kernkompetenzen“, einen 
Kandidaten ,schlussendlich irgendwie aus einer Gruppe heraus zu picken, wo man sagt, ,der verhebt' [Schweizer Dialekt für ,der wird sich bewähren']“, jemanden, den man ,verantworten (könne), den in die Unternehmen zu senden“. $\mathrm{Ob}$ sich ein Jugendlicher künftig im ,System' bewähren werde, darüber entscheide ein „gutes Bauchgefühl“ (BB-Koordinator FaBe), das man von ihm habe. Der BB-Koordinator Informatik führt in einer längeren Passage anschaulichen aus, was die spezifische Anforderung des ,Systems' Ausbildungsverbund an die Lernenden konkret bedeutet:

„Dort [im Vorstellungsgespräch] weist es sich ein bisschen, ist das jemand, mit dem wir zusammen arbeiten können oder nicht. Und da müssen wir halt schon sagen, die Leute, die in einer Verbundslösung eine Lehre machen, die müssen ein bisschen spezielle Kriterien erfüllen. Und zwar geht es dort nicht darum, wie wenn ich eigene Lernende anstelle für meinen Betrieb, (und mich frage), sehe ich diesen in meinem Betrieb?'. Sondern: ,Sehe ich den in diesen Einsatzbetrieben, die wir haben?' Und ist das möglich, dass wir dem das zumuten können, dass der jedes Jahr in einem anderen Unternehmen arbeitet, ist die Flexibilität da? Ist das nicht ein Typ, der ein bisschen Geborgenheit braucht und ein bisschen ein zurückhaltender Typ ist, wo man (...) eine Beziehung aufbauen muss, bis er aus sich heraus kommt? Das ist in einem Verbund fast nicht möglich. Das sind schon eher so ein bisschen die, ich sage jetzt mal, die Aufgeweckteren, was das Verhalten anbelangt, was aber nicht heißen muss, dass sie dann schulisch gut sind“" (BB-Koordinator Informatik).

Der zitierte BB-Koordinator grenzt die ,Passung in den Ausbildungsverbund' (Flexibilität, Offenheit) von der einzelbetrieblichen Passung ab. ,Zurückhaltendere' Jugendliche bräuchten, in den Worten des BB-Koordinators FaBe, ,einen stabileren Rahmen“, der „die Bindung an einen Betrieb“ gestatte, was das Verbundsystem nicht erlaubt. Das System lasse zudem, so der BB-Koordinator Informatik an anderer Stelle, ,null Spielraum für Leute, die sich irgendwie verschlüfe (Schweizer Dialekt für ,sich unsichtbar machen') wollen“, denn für solche Personen seien die Kontrollmöglichkeiten in einem LBV unzureichend. „Wenn das natürlich einer ist, der will, könnte er den Ausbildner und mich ausspielen jeden Tag (...). Solche Leute können wir nicht brauchen“. Damit sind die organisatorisch-räumliche Distanz zwischen LO und Ausbildungsbetrieb und die damit zusammenhängenden Probleme bei der Betreuung der Lernenden angesprochen. Die aussichtsreichsten Kandidaten für die Ausbildungsplätze sind entsprechend die vertrauenswürdigeren und ,aufgeweckteren“ unter ihnen. Dies müssen nicht ausschließlich schulisch gute Kandidaten sein, womit das Selektionskriterium Schulleistung, welches in den vorausgegangenen zwei Selektionsstufen noch hervorgehoben wurde, relativiert wird. 
Mit der geforderten Kompetenz, sich immer wieder neuen sozialen Gegebenheiten anzupassen, also Sozialbeziehungen projektförmig zu leben, beziehen sich die Repräsentanten der LO auf die ,projektbasierte' Konvention der Handlungskoordination, welche von einer projektförmigen Sozialstruktur der Arbeitswelt ausgeht. Das Äquivalenzprinzip für die Beurteilung und den Vergleich von Personen in der projektförmigen Welt stellt gemäß Boltanski (2007) die Aktivität dar, sich in von anderen initiierten Projekten einzubringen, die eigene Isolierung zu überwinden und neue Begegnungen mit anderen Akteuren einzugehen. ,Qualität' hat in dieser Welt, wer sich als anpassbar und flexibel erweist und mit ganz unterschiedlichen Situationen klar kommt. Vom Ausschluss durch die Ausbildungsorganisation LBV gefährdet sind dagegen jene Lernende, die nicht nach neuen Kontakten suchen, ,die sich nicht einzubringen wissen, weil sie kein Vertrauen erwecken, die nicht kommunizieren können, weil sie verschlossen sind“, was ,in der schönen neuen Netzwerkwelt dem sozialen Tod gleichkommt“" (ebd., Abschnitt 8).

\subsection{Kurzpraktikum im Verbundbetrieb}

Reüssieren die Kandidaten im Vorstellungsgespräch, werden sie schließlich von den BB-Koordinatoren für ein ein- bis fünftägiges „Bewerbungsschnuppern“ in ausgewählte Verbundbetriebe geschickt, wobei die BB-Koordinatoren eine möglichst realistische und Erfolg versprechende Passung vorwegnehmen. Einem Betrieb werden wenn möglich jeweils zwei Kandidaten zugeteilt, um ihm faktisch noch eine Auswahlmöglichkeit zuzugestehen. ${ }^{9}$ Bemerkenswerterweise erhalten die Ausbildner keine Bewerbungsunterlagen zur Einsicht (,ich kenne die Schulnoten nicht, ich weiß die Vorgeschichte nicht“", Ausbildnerin Altersheim), das heißt die Kandidaten werden ausschließlich auf Grundlage von Beobachtungen und Erfahrungen während des Kurzpraktikums beurteilt. Sobald ein Verbundbetrieb findet, dass ein Bewerber für die Ausbildung geeignet ist, schließt die LO mit Letzterem einen Ausbildungsvertrag ab.

Die interviewten Ausbildner in den Verbundbetrieben gaben zu Protokoll, dass sie im Rahmen dieses Kurzpraktikums v.a. darauf achten würden, ob ein Kandidat sozial in den Betrieb passe bzw. „Teamfähigkeit“ unter Beweis stelle. Dabei evaluiert die Ausbildnerin einer Kindertagesstätte auch „das allgemeine Auftreten, also die Freundlichkeit“, sowie die richtige Balance von „Nähe und Distanz" im Erstkontakt mit den zu betreuenden Kindern. Dem Ausbildner eines Behindertenheims ist wichtig, dass sich eine Bewerberin authentisch gibt und „sich wohl fühlt“. Damit beziehen sich die Ausbildner auf Anforderungen der

\footnotetext{
${ }^{9}$ Bei der Zuweisung von Lernenden ab dem zweiten Ausbildungsjahr im Rahmen der Rotation wird ihnen jeweils nur noch ein einziger Kandidat empfohlen.
} 
,häuslichen Welt', deren Erfüllung durch die Bewerber sie in der Regel affektiv beurteilen (Imdorf 2008, 2010b). Der für die Elektropraktiker zuständige Ausbildner formulierte es so: „Und wenn wir das Gefühl haben, ,das kann gehen” und es ist nichts Spezielles, wo man sagen müsste, ,Nein', dann ist es o.k.“

Die ,Schnuppertage' bieten den Ausbildern in den Betrieben zudem die Möglichkeit, Interesse, Motivation und Arbeitstugenden der Jugendlichen zu beurteilen: „Zeigt sie Motivation, Interesse, habe ich das Gefühl, diese Frau würde das gerne machen (...); sieht sie die Arbeiten?" (Ausbildnerin Kindertagesstätte). Die Ausbildner FaBe prüfen im Rahmen der Schnuppertage schließlich noch einmal, ob die Jugendlichen die für diese Dienstleistungsberufe erforderlichen Sozialkompetenzen mitbringen. In den Altersheimen wird abgeklärt, ob die künftige Lernende „mit älteren Leuten Kontakt aufnehmen kann“, und in einigen Heimen werden ältere Jugendliche den direkten Schulabgängern vorgezogen, um einer Überforderung von Lernenden und den zu betreuenden Personen vorzubeugen.

Die beschränkte Wahlfreiheit der Betriebe führt immer wieder dazu, dass die Ausbildner in den Betrieben Kompromisse eingehen, und auch Auszubildende akzeptieren, die im Selektionsprozess eines Einzelbetriebs kaum Chancen auf einen Ausbildungsplatz gehabt hätten. Sie begründen die Risikobereitschaft, auch jemanden aufzunehmen, der ,weniger in Frage kommt“ und „,von denen man nicht so genau weiß, was einem im Sommer erwartet", mit dem Argument, dass sie im Rahmen der Rotation die Lernenden jeweils nur für ein Jahr im Betrieb haben:

„Aber eh, ich denke, der Unterschied ist noch, man sagt: Ein Jahr. Ein Jahr ist machbar, auch wenn es überhaupt nicht geht, wo ich jetzt bei einer Dreijahresausbildung schon noch anders hinschaue“" (Ausbildnerin einer Kindestagesstätte).

Auch der Geschäftsführer des LBV weist darauf hin, dass die Betriebe durch den einjährigen Ausbildungsmodus Verantwortung abgeben können: „Sie [die Ausbildner] sagen sich: ,Ja, wir haben eine gewisse Verantwortung für das Jahr'. Aber ob dieser Mensch sich entwickelt, (...) ob er die Lehrabschlussprüfung besteht, das ist am Schluss (...) unser [d.h. der LO] Problem“. Die Ausbildnerin eines Altersheims verwies in diesem Zusammenhang auf das Betreuungs- und Unterstützungsangebot der LO, das es ihr ermögliche, ,unvoreingenommen junge Menschen zu betreuen“. Denn ,wenn es Probleme gibt, kann ich mich ja an sie [die BB-Koordinatoren] wenden. (...) Wenn man einem jungen Menschen eine Chance geben will, bei welchem man sagt: ,Ja, wir wissen nicht so genau', ist mir das auch recht". Auch die Ausbildnerin einer Kindertagesstätte verwies darauf, dass die höhere Akzeptanz gegenüber ,Risikojugendlichen' auch solchen 
Leuten eine Chance geben würde, denen man ,vielleicht auf den ersten Moment nicht gerade zusagen würde“.

Der Rotationsprozess selbst hat einen gewissen Selektionscharakter, da die Lernenden sich auch bei jedem Wechsel im Rahmen eines eintägigen Schnuppertages am neuen Ort vorstellen und bewähren müssen. Die Betriebe haben auch hier ein Veto-Recht, das sie jedoch selten in Anspruch nehmen. Ausbildner Elektropraktiker: „Wenn sie mir einen ganz schrägen Vogel schicken [lacht], dann kann ich auch sagen, dort, ,Nein'. Das ist aber eher selten“. Die LO sucht in diesen Fällen für den Lernenden einen alternativen Betrieb innerhalb des LBV.

Die eingeschränkte Wahlmöglichkeit der Ausbildner in den Ausbildungsbetrieben auf der letzten Stufe des Selektionsverfahrens und bei den Rotationen in Kombination mit der zeitlich eingeschränkten Verantwortung für die Lernenden sowie der Aussicht, bei Problemfällen auf die Unterstützung der BBKoordinatoren zählen zu können, mindert die betriebliche Problemantizipation und Risikoaversion in der Konfrontation mit Jugendlichen, die dem Betrieb im ersten Moment nur wenig vertrauenswürdig erscheinen. Dies erleichtert es, vermehrt staatsbürgerliche Prinzipien bei der Auswahl des Lernenden zuzulassen, ohne sie frühzeitig betrieblichen Kosten-Nutzen-Kalkülen opfern zu müssen.

\subsection{Beurteilung und Manipulation des Auswahlverfahrens durch die Verbundbetriebe}

Das präsentierte Selektionsverfahren wird von den Ausbildungsbetrieben ambivalent, aber tendenziell positiv gewürdigt. Mit Bezugnahme auf die industrielle Konvention sieht die Ausbildnerin einer Kindertagesstätte auch hinsichtlich der alljährlichen Rotation klar die Vorteile des Verfahrens: „Es ist gut, es ist ein kleiner Zeitaufwand, also ich habe diese Person einen Tag, ich muss nicht zehn Leute anschauen, die irgendwie eine Woche schnuppern kommen. Es erspart mir extrem viel Zeit. Ich muss das nicht organisieren, also die wird mir zugeteilt“ (Ausbildnerin Kindertagesstätte).

Die weitgehende Akzeptanz der eingeschränkten Wahlfreiheit während der ganzen Lehrzeit setzt Vertrauen der Ausbildner in die Vorauswahl durch die LO voraus. Ausbildner Elektropraktiker: „Also sie machen schon eine relativ gute Vorselektion eigentlich“. Ausbildnerin Altersheim: „Da ich der Überzeugung bin, dass sie ein wirklich seriöses Auswahlverfahren machen, denke ich, muss ich schon Gründe haben, um ,Nein' zu sagen“. Ausbildner Behindertenheim: „Die kommen, stellen sich vor einen Tag, wir nehmen sie und das hat bis jetzt so geklappt“. Nur der Ausbildner Informatik bemängelt, dass das schulische Leistungsniveau der zugewiesen Kandidaten z.T. zu wünschen übrig ließe. Gemäß 
Aussagen des Geschäftsführers haben die Firmen ein solches Vertrauen entwickelt, und sie wüssten, ,dass ich ihnen nicht irgendjemanden (schicke), wo sie sich nachher an den Kopf langen“ und sagen müssten, ,,, mein Gott, sind die von allen guten Geistern verlassen?'“. Die Aufrechterhaltung dieses Vertrauens ist eine delikate Angelegenheit, in der verschiedene Faktoren (Wie entwickelt sich der Lernende? Passt er zukünftig in einen Betrieb? Wie reagiert ein Betrieb auf Probleme mit einem Lernenden? etc.) hineinspielen.

Es gibt vereinzelt Betriebe, die lieber selbst selektieren würden, um ganz sicher gehen zu können, einen für den Betrieb und Beruf geeigneten Lernenden zu erhalten. Kritik wird teilweise an der Kürze des ,Schnupperpraktikums' laut, das kaum genug Zeit ließe, eine Lernende kennen zu lernen und deren Eignung vor Ort abzuklären. Gleichzeitig zeigen die Betriebe Verständnis für die Herausforderung der LO, den Pool von Lernenden im Rahmen der Endselektion sowie der Rotation auf die diversen Verbundbetriebe zu verteilen. Die Ausbildner in den Betrieben sehen ein, dass die Organisation dieser Zuteilung bei Selektion und Rotation - eine Herausforderung für die LO in der industriellen Welt - kaum machbar wäre, wenn sie bei der Auswahl zu stark mitreden würden. Aufgrund der mehrheitlich positiven Erfahrungen mit der Vorselektion des LBV wird dieser Sachzwang akzeptiert, was es möglich macht, auch einmal einen Lernenden einzustellen, der im ersten Moment nicht sonderlich überzeugt.

Dennoch kommt es in Einzelfällen vor, dass insbesondere die sozialen Institutionen (Ausbildung $\mathrm{FaBe}$ ) das Selektionsverfahren des Ausbildungsverbundes unterwandern, indem sie bereits aus früheren Praktika ${ }^{10}$ bekannte Jugendliche einzuschleusen versuchen:

Ausbildner Behindertenheim: „Ich brauche gute Lernende, ich brauche, ich will selber aussuchen, wer es ist (...). Und ich hab es jetzt so gemacht, (ich habe) mir nicht dreinreden lassen, habe Praktikanten, nächstes Jahr melde ich die an, und ,ihr [ LO] habt sie zu nehmen'“.

Das Kalkül in diesen Fällen ist, dass mit der Einstellung von schon bekannten und erfahrenen Lernenden eine Risikominimierung sowie ein zusätzlicher Profit für den Ausbildungsbetrieb im ersten Ausbildungsjahr erreicht wird. Der Geschäftsführer kritisiert dies, da sich in solchen Fällen oft zeige, ,dass die Persönlichkeit schon vorhanden ist aber schulisch weit und breit nichts da ist. Dann müssen wir halt auch nein sagen. Wenn wir dann nein sagen, dann sagt der Betrieb, wir machen nicht mit.“

${ }^{10}$ Eine in sozialen Berufen verwurzelte Tradition, an der Tätigkeit interessierte Jugendliche und junge Erwachsene zuerst als Praktikant/innen zu beschäftigen, bevor diese in ein Ausbildungsverhältnis eintreten können. 
Der Vermerk, dass die Durchsetzung der offiziellen Selektionsordnung die Ausbildungs- und Kooperationsbereitschaft einzelner Verbundbetriebe gefährden könne, verweist auf die marktförmigen Sachzwänge eines LBV. Bei der Zuweisung von Kandidaten und Lernenden geht die LO im Rahmen des Möglichen deshalb auf betriebliche Präferenzen ein, was eine gute Kenntnis betrieblicher Bedürfnisse und Eigenheiten voraussetzt. Dies ist im ganzen Passungsprozess zwischen Lernenden und Ausbildungsbetrieben während der Lehrzeit virulent. Die BB-Koordinatorin FaBe hob das matching von Lernenden und Betrieb in der häuslichen sowie in der industriellen Welt hervor: „Ich guck so ein bisschen auf die Persönlichkeit des Lernenden. Ich guck, was hat er für einen Hintergrund, passt er von der Persönlichkeit da (in den Betrieb) rein. Auch so vom Niveau her, wollen die jetzt jemand, der sehr schnell ist, oder verträgt der Betrieb (jemanden), der ein bisschen langsamer oder noch nicht so reif (ist), und dann wird das eben so gepasst“. An einer anderen Stelle benennt sie anschaulich die ,Pufferfunktion' der LO zwischen den staatsbürgerlichen Ansprüchen der Trägerschaft und der marktförmigen Nachfrage der Verbundbetriebe:

„Wir stehen so im Spannungsfeld zwischen Lehrbetrieben, Lernenden, Stadt (Name), Laufbahnzentrum, und müssen da auch ziemlich gut allen gerecht werden. Und natürlich haben wir den Fokus auf die Lernenden, aber gleichzeitig wollen wir die Betriebe nicht verlieren“.

\section{Schlussfolgerungen}

Ausbildungsverbünde gewährleisten nicht nur zusätzliche Ausbildungsplätze. Sie ermöglichen darüber hinaus, diese chancengerechter zu verteilen als dies traditionelle Betriebe bisher in der Lage waren - dies ist zumindest die hier vertretene Hypothese. LBV könnten damit neben der Stabilisierung des dualen Systems der Berufsausbildung in Zeiten der Globalisierung und Flexibilisierung auch zu mehr Chancengerechtigkeit bei der Ausbildungsplatzvergabe beitragen.

Mittels der Fallanalyse eines Ausbildungsverbundes haben wir aufgezeigt, wie sich dessen Organisationsform auf die Selektion von Auszubildenden in das Ausbildungsverhältnis eines Lehrbetriebsverbundes auswirkt. Die Rekonstruktion der Mechanismen der Selektion im Rahmen des vierstufigen Selektionsverfahrens verweist darauf, dass auf jeder Stufe unterschiedliche ausbildungsrelevante Merkmale beurteilt werden, die sich jeweils auf spezifische Konventionen der Handlungskoordination (Motive der Selektion) beziehen. In einem ersten Schritt (Vorselektion) werden die Bewerbungsdossiers einer Beurteilung nach antizipierten Effizienzkriterien der Berufsschule und der Betriebe sowie nach staatsbürgerlichen Kriterien der Benachteiligtenförderung unterzogen. Im Rah- 
men von standardisierten internen Eignungstests werden anschließend das berufliche Interesse der Kandidaten sowie (wiederholt) schulische Voraussetzungen der Ausbildung getestet. Anlässlich einer dritten Selektionsstufe klären sodann die Berufsbildungskoordinatoren in Vorstellungsgesprächen die Passung der Kandidaten in das System eines Ausbildungsverbundes ab. Relevant wird dabei die Kompetenz, Sozialbeziehungen projektförmig leben zu können, da dies im Rahmen der jährlichen Rotation zwischen den Verbundbetrieben verlangt wird. Hier handelt es sich um ein für Ausbildungsverbünde spezifisches Selektionskriterium, dem eine projektbasierte Konvention der Ausbildungsorganisation zugrunde liegt. Die vorwiegend auf Anforderungen der industriellen, projektförmigen und staatsbürgerlichen Welt ausgerichteten Bewährungstests der ersten drei Selektionsstufen verhindern, dass sich im Auswahlverfahren frühzeitig diskriminierende Kalküle der häuslichen und marktförmigen Welt eines Ausbildungsbetriebs (jene der betrieblichen Sozialintegration sowie der Kundenbindung) durchsetzen, wie das bei der Vergabe von Ausbildungsstellen in Kleinund Mittelbetrieben beobachtet werden kann. Erst auf der vierten Selektionsstufe, im Rahmen eines Kurzpraktikums im Verbundbetrieb, erhält dieser die Gelegenheit, die soziale Passung einer Kandidatin im Hinblick auf die eigenen betrieblichen Bedürfnisse zu beurteilen.

Dabei führen mehrere organisationale Mechanismen dazu, dass die sozialen Präferenzen der Verbundbetriebe nur bedingt selektionsrelevant werden. Folgende Merkmale der Ausbildungsplatzvergabe ermöglichen es einem LBV, der stark in die staatsbürgerliche Welt eingebunden ist, Diskriminierung bei der Einstellung von Lernenden einzuschränken: Erstens wird den Verbundbetrieben mit der auferlegten Entscheidung zwischen bloß zwei vorselektierten Kandidaten nur noch eine beschränkte Wahlfreiheit und Diskriminierungsmöglichkeit zugestanden. Aus Sicht der Leitorganisation handelt es sich bei der Zuweisung der Lernenden in die Betriebe - außer ein Kandidat führt sich unhaltbar auf - nur noch um ein Passungsproblem, und nicht mehr um eine Frage der Selektion. Falls ein Betrieb einen Kandidaten zurückweist, ist der LBV bestrebt, diese Person in einem alternativen Betrieb zu platzieren. Bemerkenswerterweise akzeptieren die Betriebe diese Einschränkung, denn sie zeigen für das Problem der Selektionsorganisation seitens der LO Verständnis, und sie vertrauen der LO, dass diese die Vorselektion gewissenhaft betreibt. Zweitens liegt eine klare Trennung von Vorselektion durch die LO und Endselektion durch die Betriebe vor, wobei letztere keine Kenntnis von den Bewerbungsdossiers der Kandidaten erhalten, und somit auch nicht die Möglichkeit haben, generalisierende Schlüsse von Angaben zu Nationalität, Familiensituation oder Schulzeugnissen auf die häuslichen und marktförmigen ,Qualitäten' einer Kandidatin zu ziehen. Die betriebliche Beurteilung dieser ,Qualitäten' erfolgt vielmehr auf der Grundlage eines individualisti- 
schen Praxistests (Imdorf 2010a) unter betrieblichen Realbedingungen. Auch die LO scheint bei der Beurteilung der ,System-Passung' auf kollektivistische Kriterien (Geschlecht, nationale Herkunft) weitgehend zu verzichten. Drittens senkt die auf ein Ausbildungsjahr beschränkte zeitliche Verantwortung, welche ein Verbundbetrieb für seinen Lernenden wahrnehmen muss, die Hemmschwelle gegenüber Kandidaten, die in der häuslichen oder marktförmigen Welt eines Betriebs als problematisch gelten. Denn durch das Rotationsprinzip verteilt sich das Problemrisiko eines Jugendlichen auf mehrere Betriebe. Viertens mindert auch das durch den LBV angebotene professionelle Unterstützungsangebot bei Ausbildungsproblemen mit Lernenden die Risikoaversion gegenüber Jugendlichen, die aus betrieblicher Sicht potentiell Probleme bereiten könnten. ${ }^{11}$ Fünftens bewirkt die Rotation während der Ausbildung eine ,Selektion in Raten', wobei die LO im seltenen Fall, dass ein Betrieb einen im zweiten oder dritten Lehrjahr zugewiesenen Lernenden ablehnt, verbundsintern nach einer alternativen Lösung sucht, so dass die Ausbildung nicht abgebrochen werden muss.

Eine Vorselektion, die von einer frühzeitigen Ausbeutung sozialer Kategorien absieht, erinnert an das Selektionsverfahren eines Großbetriebes, das auf die Ermöglichung sozialer Vielfalt (diversity) ausgerichtet ist. Bemerkenswert ist, dass dieses Verfahren im Fall des untersuchten LBV Kleinbetrieben zugute kommt, die bei traditionellen Selektionsprozessen in der Regel eher soziale Homogenität reproduzieren. Die Verschiebung des organisationalen Passungskriteriums von der „Betriebstauglichkeit“ zur „Verbundtauglichkeit“ ermöglicht es dem untersuchten LBV entsprechend, seinen Sozialauftrag zu erfüllen und benachteiligte Jugendliche an solche Verbundbetriebe zu vermitteln, die für das soziale Anliegen nicht notgedrungen sensibilisiert sein oder in diese Richtung Interessen haben müssen. Selbst wenn die betrieblichen Anforderungen der häuslichen und marktförmigen Welt weiter bestehen, so wird die Durchsetzbarkeit ihrer Selektionskalküle durch die aufgeführten organisationalen Mechanismen geschwächt. Es kann aber auch sein, dass einige Verbundbetriebe ihre Organisationskultur durch die positive Erfahrung mit einer ehemals problematisierten Kategorie von Jugendlichen dahingehend verändern, dass sie sich künftig offener für die Ausbildung solcher Personen zeigen (vgl. Eingangszitat).

Der Preis des egalitären Selektionsverfahrens eines LBV ist jedoch womöglich die Produktion einer neuen Form der Benachteiligung: ,Verbundtauglichkeit' verlangt von den Jugendlichen Flexibilität und die Kompetenz, soziale Beziehungen projektförmig zu leben. Wer Mühe damit hat, dauernd neue Kontakte einzugehen und alte aufzugeben, ist potenziell vom Ausschluss aus der

${ }^{11}$ Beide Faktoren der reduzierten betrieblichen Risikoaversion, die nur einjährige Ausbildungsdauer pro Betrieb sowie das Unterstützungsangebot bei Ausbildungsproblemen, dürften die Folge davon sein, dass die Betriebe eines LBV von der Verantwortung für den Ausbildungserfolg befreit sind. 
Netzwerkwelt eines Ausbildungsverbundes betroffen. Inwiefern dadurch eine neue Form sozialer Ungleichheit entsteht, bleibt eine drängende Forschungsfrage.

Zum Schluss bleiben zwei weitere Forschungsdesiderate: Zum einen steht der Beweis aus, dass ein LBV wie der von uns untersuchte durch die oben genannten Selektionsmechanismen tatsächlich weniger Diskriminierung erzeugt, als dies bei der Einstellung von Lernenden in traditionellen Ausbildungsbetrieben der Fall ist. Auch wenn die Aussagen der Interviewpartner für die Annahme sprechen, dass LBV ihre Ausbildungsplätze kraft ihrer Ausbildungsorganisation egalitärer vergeben können als konventionelle Ausbildungsbetriebe (in den Interviews wurde vermerkt, dass die Lernenden meist das Übergangssystem beansprucht hätten, überdurchschnittlich alt seien, oft ,aus anderen Kulturen“ kämen, und teilweise „leichte Behinderungen“ hätten), so bleibt ein statistisch erhärteter Beweis für unsere Hypothese zur Zeit noch aus. Zum anderen bleibt zu prüfen, ob auch Ausbildungsverbünden ohne Sozialauftrag, die ihre Selektion in ähnlicher Weise wie der untersuchte LBV organisieren, die Integration von benachteiligten Schulabgängern besser gelingt als traditionellen Ausbildungsberufen. Es könnte sein, dass in ausschließlich selbstfinanzierten Ausbildungsverbünden ohne staatliche oder parastaatliche Trägerschaft bei der Selektion von Lernenden die marktwirtschaftliche Konvention des LBV eine größere Bedeutung entwickelt und vermehrt einzelbetriebliche, mitunter diskriminierende Kalküle mitberücksichtigt werden müssen, da die finanzielle Abhängigkeit der LO von den Verbundbetrieben größer ist.

\section{Literatur}

BBT (Bundesamt für Berufsbildung und Technologie) (2008): Resultate Evaluation Lehrbetriebsverbünde. Bern.

Becker, G. S. (1971): The Economics of Discrimination. Second Edition. Chicago.

BMBF (Bundesministerium für Bildung und Forschung) (2004): Berufsbildungsbericht 2004. Bonn/Berlin.

Boltanski, L. (2007): Leben als Projekt. Prekarität in der schönen neuen Netzwerkwelt. Polar (2). S. 7-13.

Boltanski, L. / Chiapello, E. (2003): Der neue Geist des Kapitalismus. Konstanz.

Boltanski, L. / Thévenot, L. (1999): The Sociology of Critical Capacity. European Journal of Social Theory, 2(3). S. 359-377.

Boltanski, L. / Thévenot, L. (2007): Über die Rechtfertigung. Eine Soziologie der kritischen Urteilskraft. Hamburg.

DBK, DBK-Projektgruppe Verbundkonzepte (2002): Handbuch für Ausbildungsverbünde. Schweizerisches Dienstleistungszentrum Berufsbildung. Bern. 
Diaz-Bone, R. (2009): Konvention, Organisation und Institution. Der institutionentheoretische Beitrag der ,Économie des conventions‘. Historical Social Research, 34(2). S. 235-264.

Dodier, N. (2010): Konventionen als Stützen der Handlung. Elemente der soziologischen Pragmatik. Trivium, 3 (5-2010). [En ligne], mis en ligne le 10 janvier 2010. URL : http://trivium.revues.org/index3565.html. Consulté le 4 juin 2010.

Eymard-Duvernay, F. / Marchal, E. (1997): Façons de recruter. Le jugement des compétences sur le marché du travail. Paris.

Imdorf, C. (2008): Migrantenjugendliche in der betrieblichen Ausbildungsplatzvergabe auch ein Problem für Kommunen. In: Bommes, M. / Krüger-Potratz, M. (Hrsg.): Migrationsreport 2008. Fakten - Analysen - Perspektiven. Frankfurt a. M. S. 113158.

Imdorf, C. (2009): Die betriebliche Verwertung von Schulzeugnissen bei der Ausbildungsstellenvergabe. Empirische Pädagogik, 23 (4). S. 392-409.

Imdorf, C. (2010a): Die Diskriminierung, ausländischer' Jugendlicher bei der Lehrlingsauswahl. In: Hormel, U. / Scherr, A. (Hrsg.): Diskriminierung. Grundlagen und Forschungsergebnisse. Wiesbaden. S. 197-219.

Imdorf, C. (2010b): Emotions in the hiring procedure: How 'gut feelings' rationalize personnel selection decisions. In: Wettergren, $\AA$ / Sieben, B. (Hrsg.): The feeling organization. New York/Houndmills. In print.

Imdorf, C. (2010c): Wie Ausbildungsbetriebe soziale Ungleichheit reproduzieren: Der Ausschluss von Migrantenjugendlichen bei der Lehrlingsselektion. In: Krüger, H.-H. / Rabe-Kleberg, U. / Kramer, R.-T. / Budde, J. (Hrsg.): Bildungsungleichheit revisited. Bildung und soziale Ungleichheit vom Kindergarten bis zur Hochschule. Wiesbaden. S. 263-278.

Jenkins, R. (1984): Acceptability, Suitability and the Search for the Habituated Worker: How Ethnic Minorities and Women Lose Out. International Journal of Social Economics, 11 (7). S. 64-76.

Jewson, N. / Mason, D. (1986): Modes of Discrimination in the Recruitment Process: Formalisation, Fairness and Efficiency. Sociology, 20 (1). S. 43-63.

Moser, U. (2004): Jugendliche zwischen Schule und Berufsbildung. Eine Evaluation bei Schweizer Grossunternehmen unter Berücksichtigung des internationalen Schulleistungsvergleich PISA. Bern.

Reskin, B. F. (2003): Including Mechanisms in Our Models of Ascriptive Inequality. American Sociological Review, 68 (February). S. 1-21.

Schmid, M. / Storni, M. (2004): Transition. Zur Qualifikation und Rekrutierung von Lehrlingen im Kanton Basel-Stadt (Schlussbericht des Forschungsprojektes Transition). ecce gemeinschaft für sozialforschung. Basel.

Schweri, J. / Mühlemann, S. / Pescio, Y. / Walther, B. / Wolter, S. C. / Zürcher, L. (2003): Kosten und Nutzen der Lehrlingsausbildung aus der Sicht Schweizer Betriebe. Zürich.

Sehringer, R. (1989): Betriebliche Strategien der Personalrekrutierung. Ergebnisse einer Betriebsbefragung. Frankfurt a. M. 
Seibert, .H. / Hupka, S. / Imdorf, C. (2009): Wie Ausbildungssysteme Chancen verteilen Berufsbildungschancen und ethnische Herkunft in Deutschland und der Schweiz unter Berücksichtigung des regionalen Verhältnisses von betrieblichen und schulischen Ausbildungen. Kölner Zeitschrift für Soziologie und Sozialpsychologie, 61 (4). S. 595-620.

Stalder, B. (2000): Gesucht wird... Rekrutierung und Selektion von Lehrlingen im Kanton Bern. Amt für Bildungsforschung. Bern.

Walther, B. / Renold, U. (2005): Lehrbetriebsverbund - neue Chancen für Klein- und Mittelbetriebe. Die Volkswirtschaft, 2005 (4). S. 39-42.

Yin, R. K. (2009): Case Study Research. Design and Methods. Fourth Edition. Thousand Oaks. 